\title{
Higher Psoriasis Skin Clearance Is Associated with Lower Annual Indirect Costs in the United States: A Post Hoc Analysis from the CLEAR Study
}

Steven R. Feldman, MD, PhD; Yang Zhao, PhD; Isabelle Gilloteau, MSc, MPH; Christopher N. Graham, MS; LaStella Miles, MS; Doreen McBride, PhD, MBA; and Vivian Herrera, DDS, MPH

\begin{abstract}
BACKGROUND: Psoriasis is associated with a high economic burden to society. New psoriasis systemic treatments offer the potential for improved skin clearance. Whether a higher degree of clearing translates into economic benefit through decreased work impairment has not been fully determined.

OBJECTIVE: To assess whether more complete clearing of psoriasis is associated with a reduction in disease-related indirect costs.

METHODS: Pooled data from employed patients included in the CLEAR study, a phase $3 \mathrm{~b}$ study comparing the efficacy and safety of secukinumab (337 subjects) versus ustekinumab (339 subjects), were classified into 4 levels of skin clearance improvement at weeks 16 and 52: Psoriasis Area and Severity Index (PASI) improvement from baseline of $<50 \%(\mathrm{PASI}<50)$, $50 \%-74 \%$ (PASI $50-74$ ), $75 \%-89 \%$ (PASI $75-89$ ), and $\geq 90 \%$ (PASI $\geq 90$ ). Patients completed the Work Productivity and Activity Impairment questionnaire for psoriasis (WPAI-PS0), which assessed absenteeism, presenteeism, and a composite overall work impairment over the previous 7 days at weeks 16 and 52. U.S. Department of Labor data were used to calculate annual indirect costs due to work productivity loss.
\end{abstract}

RESULTS: In the CLEAR study, 452 (67\%) were employed at baseline and included in this analysis. At week 16, mean overall work impairment significantly decreased with higher PASI improvements: $22.8 \%$ for $\mathrm{PASI}<50$, compared with $13.3 \%$ for PASI $50-74(P=0.001) ; 6.4 \%$ for PASI $75-89$ $(P<0.001)$; and $4.9 \%$ for $P A S I \geq 90(P<0.001)$, with the majority of work impairment related to presenteeism. Calculated mean work hours lost by overall work impairment decreased with higher PASI improvements: 8.2 hours lost/week (429 hours/year) for patients with PASI 50; 4.6 hours lost/ week (251 hours/year) for PASI 50-74; 2.3 hours lost/week (121 hours/ year) for PASI 75-89; and 1.8 hours lost/week (93 hours/year) for PASI $\geq 90$. Associated mean annual indirect costs due to work productivity loss per worker decreased with higher PASI improvements: $\$ 10,318$ for $\mathrm{PASI}<50$, $\$ 6,042$ for PASI 50-74, $\$ 2,901$ for PASI 75-89, and $\$ 2,233$ for PASI $\geq 90$. Similar results were observed at week 52 . Mean overall work impairment decreased with higher PASI improvements, ranging from $26.3 \%$ for $\mathrm{PASI}<50$ to $6.9 \%$ for $P A S I \geq 90$. A decrease in overall work hours lost (ranging from 9.5 hours lost/week [ 495 hours/year] for PASI $<50$ to 2.5 hours/week [130 hours/year] for PASI $\geq 90$ ), as well as associated annual indirect costs due to work productivity loss (ranging from $\$ 11,906$ for $\mathrm{PASI}<50$ to $\$ 3,125$ for $\mathrm{PASI} \geq 90$ ), were also shown with higher PASI improvements at week 52 .

CONCLUSIONS: Among working patients with moderate to severe psoriasis, higher PASI improvements were associated with lower work productivity loss and reduced annual indirect costs. By improving and sustaining skin clearance, psoriasis treatments may contribute to increased work productivity and decreased societal economic burden.

J Manag Care Spec Pharm. 2018;24(7):617-22

Copyright $\odot 2018$, Academy of Managed Care Pharmacy. All rights reserved.

\section{What is already known about this subject}

Psoriasis affects work productivity, including absenteeism and presenteeism, particularly in patients with moderate to severe psoriasis.

Cost of work productivity loss accounts for more than $30 \%$ of the total societal cost of psoriasis.

\section{What this study adds}

Using a clinical trial study in subjects with moderate to severe psoriasis, this analysis demonstrated that higher skin clearance is associated with a reduction in overall work productivity loss and annual work hours lost.

Decreasing absenteeism and presenteeism through better skin clearance may reduce the overall costs of psoriasis to employers and society.

D soriasis is a chronic, immune-mediated skin disease that affects approximately $2 \%-3 \%$ of the population in the United States, ${ }^{1,2}$ generally among people of working age. ${ }^{3}$ The economic burden of psoriasis to society is significant, with $62 \%$ higher direct medical costs and 50\% higher indirect costs compared with patients without psoriasis. ${ }^{4}$ The total economic burden of psoriasis in the United States in 2013 was estimated to be $\$ 35.2$ billion, with work productivity loss accounting for $32 \%$ of the total burden, or $\$ 11.2$ billion.

Among U.S. patients with moderate to severe psoriasis, there are limited data on indirect costs due to loss of work productivity (absenteeism and presenteeism [impairment while working]) by measures of treatment effect (e.g., change in Psoriasis Area and Severity Index [PASI] scores). However, increasing severity of psoriasis has a significant negative effect on work productivity, with overall work impairment among U.S. patients with severe psoriasis more than 2 to 3 times the impairment of patients with mild psoriasis. 6,7 The assessment of employment and work productivity loss alongside disease severity in long-term clinical trials allows for estimations of the effect of psoriasis treatment, stratified by skin clearance levels, on work impairment and associated costs. 


\begin{tabular}{|c|c|c|}
\hline Parameter & Value & Source \\
\hline $\begin{array}{l}\text { Percentage of part-time } \\
\text { employment, \% }\end{array}$ & 18.4 & $\begin{array}{l}\text { U.S. Bureau of Labor } \\
\text { Statistics, } 201516\end{array}$ \\
\hline \multicolumn{3}{|c|}{ Average work hours per week } \\
\hline Full time & 39.5 & \multirow{2}{*}{$\begin{array}{l}\text { U.S. Bureau of Labor } \\
\text { Statistics, } 2011^{17}\end{array}$} \\
\hline Part time & 20.7 & \\
\hline \multicolumn{3}{|l|}{ Average hourly wage, \$ } \\
\hline Full time & 25.33 & \multirow{2}{*}{$\begin{array}{l}\text { U.S. Bureau of Labor } \\
\text { Statistics, } 2011^{17} \text {; } \\
\text { U.S. Bureau of Labor } \\
\text { Statistics, 2005-201618 }\end{array}$} \\
\hline Part time & 13.50 & \\
\hline
\end{tabular}

The accepted gold standard for assessing disease severity is the PASI,${ }^{8}$ which measures the average redness, thickness, and scaliness of lesions, ranging from 0 (no disease) to 72 (maximum disease). Psoriasis severity has been defined using the following criteria: mild, PASI $<7$ and Dermatology Quality of Life Index (DLQI) $<7$; moderate, PASI = 7-15 and DLQI = 5-15 (or classified as severe when difficult-to-treat sites are affected or when there is a significant psychosocial effect); severe, PASI > 15, independent of the DLQI score. ${ }^{9}$ To evaluate the clinically meaningful success of treatment, the percentage of improvement in skin lesions is commonly used as an endpoint in clinical trials, with at least $75 \%$ improvement in the PASI score (PASI 75) demonstrating treatment success. ${ }^{10}$ However, with increased efficacy shown by newly developed treatments for psoriasis, PASI 90 (90\% improvement in disease severity) has been suggested as the new standard. ${ }^{11}$

New psoriasis treatments, particularly biologics that target interleukin (IL)-17A (secukinumab, ixekizumab) or IL-12/23 (ustekinumab), offer the potential for clearing psoriasis more completely than was possible with previous treatments. Although these treatments reduce work productivity loss, ${ }^{12}$ it has not been fully determined whether higher degrees of clearing are economically beneficial to employers and society as a whole.

The objective of this study was to assess, from clinical trial data, the association of skin clearance by change in PASI score with the effect on work impairment and associated indirect costs.

\section{Methods}

This was a post hoc analysis of data from the CLEAR study, a phase $3 \mathrm{~b}$ study comparing the efficacy and safety of secukinumab versus ustekinumab. ${ }^{13}$ The study design has been previously described. ${ }^{13}$ Briefly, CLEAR was a multicenter, randomized, double-blind, head-to-head, parallelgroup superiority trial. Subjects who were aged $\geq 18$ years and had moderate to severe plaque psoriasis (defined as PASI score $\geq 12$ and affected body surface area $\geq 10 \%$ and Investigator's Global Assessment [IGA] $\bmod 2011 \geq 3$ ) were

\section{TABLE 2 Baseline Characteristics of Employed Patients}

\begin{tabular}{l|rc}
\hline Parameter (N =452) & \multicolumn{2}{|c}{ Value } \\
\hline Age, mean (SD) & 43.0 & $(11.80)$ \\
\hline Male sex, $\mathrm{n}(\%)$ & 334.0 & $(73.90)$ \\
\hline Weight $(\mathrm{kg})$, mean (SD) & 87.4 & $(20.07)$ \\
\hline Body mass index $\left(\mathrm{kg} / \mathrm{m}^{2}\right)$, mean (SD) & 28.7 & $(5.81)$ \\
\hline Presence of psoriatic arthritis, $\mathrm{n}(\%)$ & 76.0 & $(16.80)$ \\
\hline Years since first psoriasis diagnosis, mean (SD) & $17.7 \quad(11.42)$ \\
\hline Source: Data on file. Novartis. Demographics and background characteristics of \\
employed patients in CLEAR. 2017.
\end{tabular}

eligible. Subjects had a diagnosis of psoriasis at least 6 months before randomization and had been inadequately controlled by topical treatments, phototherapy, and/or previous systemic therapy. Key exclusion criteria included previous exposure to any biologics directly targeting IL-17A/IL-17 receptor A or IL-12/IL-23.13 Eligible patients were randomized to either secukinumab $300 \mathrm{mg}$ or ustekinumab (dosage determined by body weight). Secukinumab was given at baseline and weeks $1,2,3$, and 4 , and then every 4 weeks thereafter; ustekinumab was given at baseline and week 4 , then every 12 weeks thereafter. Placebo injections were also given to match the secukinumab group to maintain blinding. Data from both treatment arms from the CLEAR trial were pooled, and only patients who were employed at baseline were included in this analysis. Psoriasis skin clearance was defined using the PASI, and effect on work was evaluated using the Work Productivity and Activity Impairment questionnaire (WPAI) at weeks 16 and $52 .{ }^{14}$ The analysis was conducted from the perspective of the indirect costs to employers in the United States.

\section{Psoriasis Area and Severity Index}

The PASI is a clinician-reported instrument used to assess the severity of psoriasis by providing weighted measurement of the average redness, thickness, and scaliness of psoriasis lesions. ${ }^{8}$ Pooled secukinumab and ustekinumab data were stratified by 4 levels of PASI score change from baseline to week 16 and baseline to week 52, respectively: improvement of less than 50\% from baseline (PASI <50), 50\%-74\% (PASI 50-74), 75\%-89\% (PASI 75-89), and at least 90\% (PASI $\geq 90$ ). Although PASI 75 response (at least $75 \%$ improvement in PASI score from baseline) is recognized as a clinically meaningful endpoint in clinical trials for the treatment of psoriasis, PASI 90 (at least 90\% improvement) has been used in the development of many psoriasis treatments and served as the primary outcome of the CLEAR trial. ${ }^{10}$ 


\section{FIGURE 1 Week 16 Absenteeism and Presenteeism by PASI Levels}

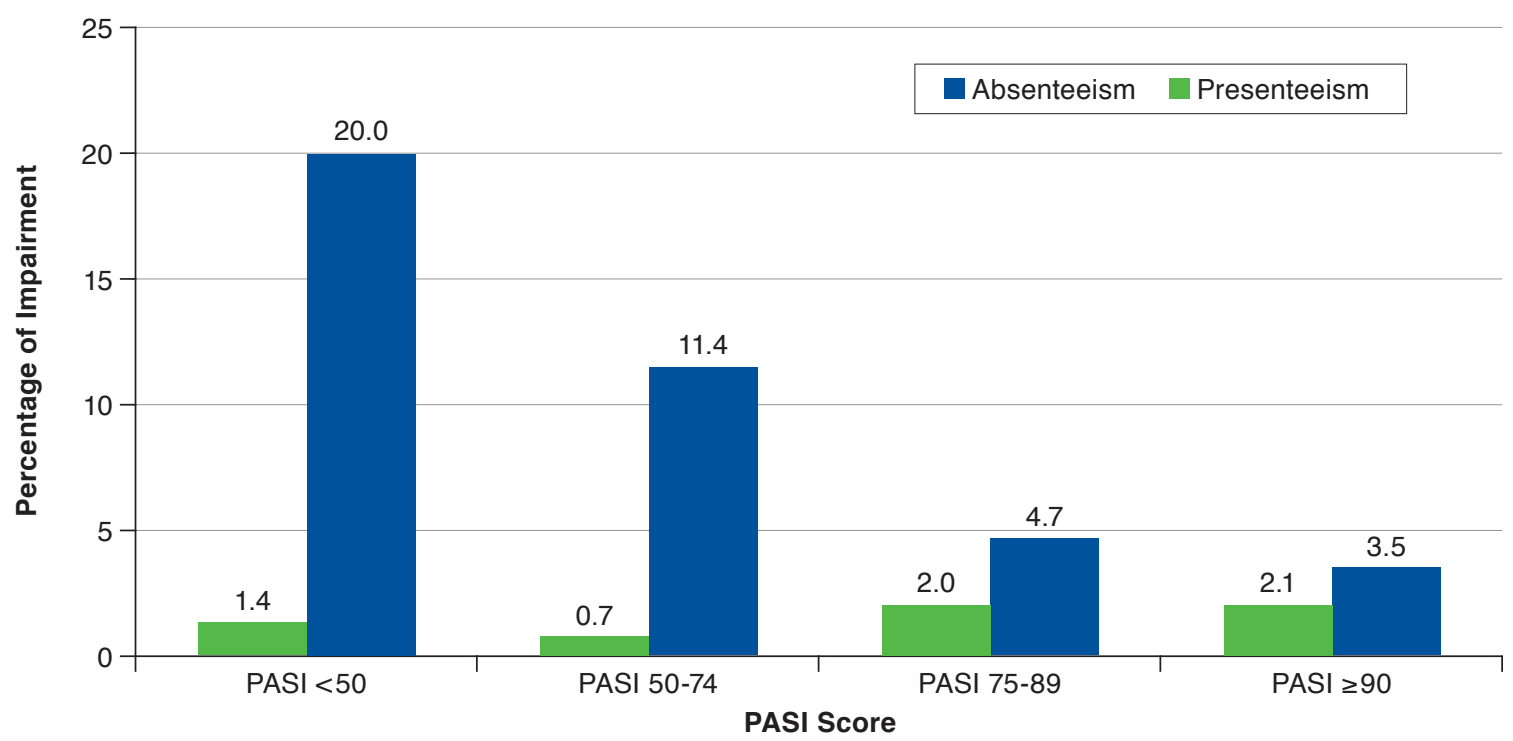

PASI = Psoriasis Area and Severity Index.

\section{Work Productivity and Activity Impairment Questionnaire}

The WPAI questionnaire is a validated quantitative instrument that assesses health-related work productivity over the previous week, encompassing 4 subdomains: the percentage of overall work impairment, work time missed (absenteeism), reduced on-the-job effectiveness (presenteeism), and daily activity impairment. ${ }^{14}$ Overall work impairment is a composite of absenteeism and presenteeism. Work impairment and activity impairment are assessed on a scale of 0 to 100, with a higher score indicating a greater level of impairment. Of the 4 subdomains, only the first 3 are relevant to patients who are currently in paid employment. The WPAI-PSO specifically targets loss of productivity due to psoriasis. ${ }^{15}$

\section{Employment Parameters and Costs}

For all trial patients employed at baseline, reported work time missed (absenteeism) and impairment while working (presenteeism) from the previous 7 days were captured by the WPAIPSO at weeks 16 and 52. WPAI-PSO scores were then used to estimate the percentage of overall work impairment due to psoriasis at the 2 time points.

Employment parameter inputs, including the current national averages for full-time and part-time employment in the United States, ${ }^{16}$ the hours worked per week, and hourly wages (most recent rates from 2010) were obtained from the U.S. Bureau of Labor Statistics. ${ }^{17}$ Wages in U.S. dollars were adjusted to 2016 by multiplying by the average 2016 inflation rate divided by the average 2010 rate (Table 1). ${ }^{18}$

\section{Statistical Analysis}

Productivity loss (average number of work hours lost per week by absenteeism and presenteeism due to psoriasis) was calculated as the average number of full- and part-time work hours per week times the percentage of work impairment for each PASI level. Statistical significance in overall work impairment between PASI $<50$ and the higher PASI levels was assessed in a pairwise fashion using one-way analysis of variance (ANOVA). No adjustments were made for multiple comparison for this exploratory analysis. Indirect costs were calculated by multiplying the weekly productivity loss by the average full- and part-time hourly wage data. The weekly hours and indirect costs were then annualized.

\section{Results}

The CLEAR study included 676 patients with moderate to severe psoriasis (secukinumab $=337$, ustekinumab $=339$ ); 452 (67\%) patients reported current employment at baseline and were included in the current analysis. ${ }^{13}$ The mean age was 43 years, and the majority of the patients were male (74\%) and overweight, with a mean body mass index of 28.7 (Table 2).

At week 16, work impairment due to psoriasis significantly decreased with greater skin clearance. Those achieving PASI $<50(n=18)$ reported an overall work impairment of $22.8 \%$ compared with $13.3 \%$ for patients with PASI 50-74 ( $n=29$; $P=0.001) ; 6.4 \%$ for those with PASI $75-89(n=89 ; P \leq 0.001)$; and $4.9 \%$ for those with PASI $\geq 90(n=168 ; P \leq 0.001)$. Overall 


\section{FIGURE 2 Week 52 Absenteeism and Presenteeism by PASI Levels}

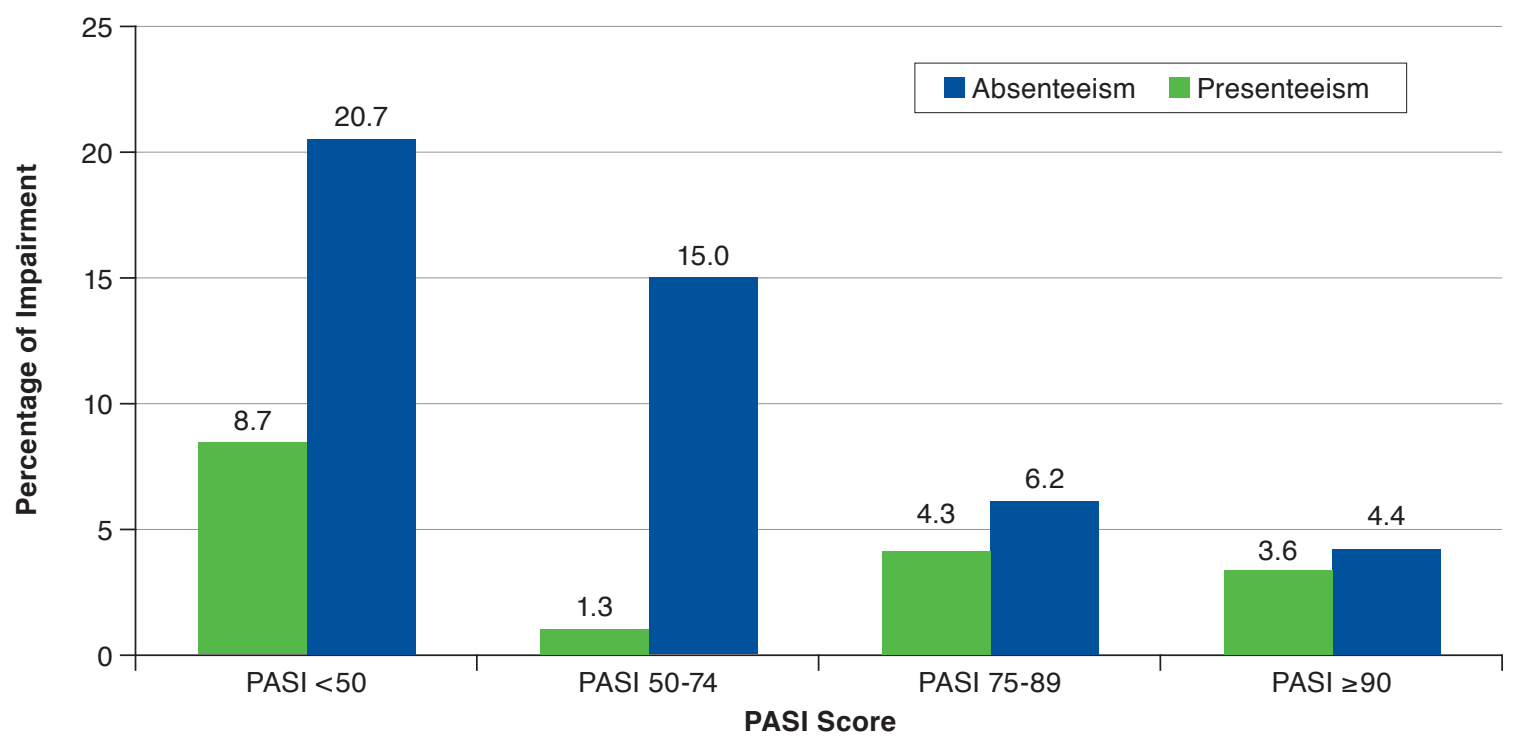

PASI = Psoriasis Area and Severity Index.

work impairment was mostly related to productivity loss at work (presenteeism) rather than to work time missed (absenteeism; Figure 1). For PASI <50, for example, there was a $20.0 \%$ loss due to presenteeism, compared with $1.4 \%$ due to absenteeism. On average, the overall productive work time missed from absenteeism and presenteeism due to psoriasis significantly decreased with increasing psoriasis skin clearance. Patients with PASI $<50$ missed a mean of 8.2 hours of work weekly (429 hours/year). The mean values for the other response rates were 4.8 hours/week (251 hours/year) for PASI 50-74; 2.3 hours/ week (121 hours/year) for PASI 75-89; and 1.8 hours/week (93 hours/year) for PASI $\geq 90$. Projected annual mean indirect costs due to work productivity loss per worker also decreased with greater PASI improvement: $\$ 10,318$ for PASI $<50 ; \$ 6,042$ for PASI 50-74; $\$ 2,901$ for PASI 75-89; and \$2,233 for PASI $\geq 90$. Indirect costs associated with $\mathrm{PASI} \geq 90$ response were $78 \%$ lower than those associated with PASI $<50$ response, 63\% lower than those associated with PASI 50-74 response, and 23\% lower than those associated with PASI 75-89 response.

Similar results were observed at week 52. Patients achieving PASI < $50(n=14)$ reported greater overall work impairment of $26.3 \%$ compared with $16.4 \%$ for patients with PASI $50-74$ $(n=30 ; P=0.102) ; 10.4 \%$ for patients with PASI $75-89(n=92$; $P=0.004)$; and $6.9 \%$ for those achieving PASI $\geq 90 \quad(n=366$; $P<0.001$ ). Consistent with the findings from week 16, work impairment at week 52 was mainly due to presenteeism, particularly with lower skin clearance (PASI $<75$; Figure 2). Patients with PASI $<50$ missed a mean of 9.5 hours/week
(494 hours/year); 5.9 hours/week (308 hours/year) for PASI 50-74; 3.7 hours/week (195 hours/year) for PASI 75-89; and 2.5 hours/week (130 hours/year) for PASI $\geq 90$. Projected mean annual indirect costs due to work productivity loss per worker decreased with greater PASI improvement $(\$ 11,906$ for PASI $<50, \$ 7,405$ for PASI 50-74, \$4,697 for PASI 75-89, and $\$ 3,125$ for PASI $\geq 90$ ). Indirect costs associated with PASI $\geq 90$ response were $74 \%$ lower than those associated with PASI $<50$ response, 58\% lower than those associated with PASI 50-74 response, and 33\% lower than those associated with PASI 75-89 response.

\section{Discussion}

Using pooled data from a phase 3 clinical trial in moderate to severe psoriasis, we evaluated the effect of the degree of skin clearance on work productivity loss and associated indirect costs. Increasing levels of skin clearance, as measured by reduction in PASI scores, led to significantly decreased rates of work impairment assessed at both weeks 16 and 52 of treatment, thereby reducing indirect costs.

Psoriasis reduces patient work productivity, with greater impacts seen with more severe disease. ${ }^{6,7}$ However, few studies have evaluated work productivity impairment using validated measures such as the WPAI within the psoriasis population or evaluated the effect of skin clearance as measured by PASI on aspects of work productivity when patients have been under treatment for longer periods of time. This analysis fills this gap in evidence. As newer therapies attain a greater response of 


\section{Higher Psoriasis Skin Clearance Is Associated with Lower Annual Indirect Costs in the United States: A Post Hoc Analysis from the CLEAR Study}

PASI 90 or higher, results from this analysis provide a way to quantify the value in increased workplace productivity and reduction in annual direct costs based on the level of skin clearance. ${ }^{19}$

Increasing disease severity reduces the ability to work full time or be employed. A U.S. survey conducted among respondents aged 30 years or older found that patients with selfreported severe disease had a lower probability of working full time than patients with self-reported mild disease, and patients who self-reported severe disease were more likely to report that psoriasis was the reason for not working. ${ }^{20}$ Korman et al. (2015) also found greater work productivity loss, absenteeism, and presenteeism in patients with physician-confirmed moderate or severe psoriasis, compared with patients with mild disease. $^{7}$ Our analysis confirms these results, using validated measurements of disease severity and work productivity. In addition, the employment rate of $67 \%$ in the study population with psoriasis used in this analysis is more than 10\% lower than that of a similarly aged general U.S. population (ages 45-49) for which an employment rate of $78 \%$ is reported. ${ }^{16}$

\section{Limitations}

There were a number of limitations to this study. Wages and proportion of full-time and part-time employment for the general population were assumed to be similar to those for the psoriasis population. The percentage of patients with psoriasis who were employed was obtained from the CLEAR trial data and may not be representative of real-world employment percentages in the entire psoriasis population. Annual costs were estimated by extrapolating based on data collected for the 1-week recall, and work impairment may vary over a longer time period. Analysis of subgroups, such as patients with psoriatic arthritis, was not conducted. The objective of this post hoc analysis using CLEAR data was not to compare the effects of secukinumab and ustekinumab on work impairment, which had been previously reported by Blauvelt et al. (2017), ${ }^{13}$ nor compare the cost of therapy versus an offset of productivity gain. The analysis of the pooled data from this trial accomplished our objective to assess the effect of increased skin clearance on work impairment. Future research should include evaluation of the effect on activities for patients who are not employed and specific subgroup analyses.

\section{Conclusions}

Among employed patients with moderate to severe psoriasis, higher skin clearance as measured by PASI was associated with a greater increase in workplace productivity and projected a reduction in annual indirect costs. These results were consistent at weeks 16 and 52 and demonstrate the benefit that treatments providing long-term levels of high PASI improvement bring to both patients of working age and society. These results indicate that by improving skin clearance, psoriasis treatments may contribute to reduced work productivity loss and substantial attendant indirect costs.

\section{Authors}

STEVEN R. FELDMAN, MD, PhD, Wake Forest University School of Medicine, Winston-Salem, North Carolina. YANG ZHAO, PhD, and VIVIAN HERRERA, DDS, MPH, Novartis Pharmaceuticals, East Hanover, New Jersey. ISABELLE GILLOTEAU, MSc, MPH, Novartis Pharma AG, Basel, Switzerland, and DOREEN MCBRIDE, PhD, MBA, RTI Health Solutions, Manchester, United Kingdom. CHRISTOPHER N. GRAHAM, MS, and LASTELLA MILES, MS, RTI Health Solutions, Research Triangle Park, North Carolina.

AUTHOR CORRESPONDENCE: Doreen McBride, PhD, MBA, RTI Health Solutions, The Pavilion, Towers Business Park, Wilmslow Rd., Didsbury, Manchester M20 2LS UK. Tel.: 919.485.2626; E-mail: dmcbride@rti.org.

\section{DISCLOSURES}

Funding for this study was provided by Novartis Pharmaceuticals. Zhao and Herrera are employed by Novartis. Gilloteau is employed by Novartis Pharma AG. McBride, Graham, and Miles are employed by RTI Health Solutions, which provides consulting and other research services to pharmaceutical, device, governmental, and nongovernmental organizations and received funding from Novartis for manuscript development, analysis development, and general consultation. Feldman reports grants and personal fees from Novartis, Abbvie, Janssen, Lilly, and Celgene, along with personal fees from Amgen and Valeant.

\section{ACKNOWLEDGMENTS}

The authors thank the patients and investigators who participated in the CLEAR trial and Bintu Sherif of RTI Health Solutions for her statistical analysis support.

\section{REFERENCES}

1. Kurd SK, Gelfand JM. The prevalence of previously diagnosed and undiagnosed psoriasis in U.S. adults: results from NHANES 2003-2004. J Am Acad Dermatol. 2009;60(2):218-24.

2.Lebwohl MG, Bachelez H, Barker J, et al. Patient perspectives in the management of psoriasis: results from the population-based Multinational Assessment of Psoriasis and Psoriatic Arthritis Survey. J Am Acad Dermatol. 2014;70(5):871-81.el-e30.

3. Raval K, Lofland JH, Waters H, Piech CT. Disease and treatment burden of psoriasis: examining the impact of biologics. J Drugs Dermatol. 2011;10(2):189-96.

4. Svedbom A, Dahlén J, Mamolo C, et al. Economic burden of psoriasis and potential cost offsets with biologic treatment: a Swedish register analysis. Acta Derm Venereol. 2016 Jun 15;96(5):651-57.

5. Vanderpuye-Orgle J, Zhao Y, Lu J, et al. Evaluating the economic burden of psoriasis in the United States. J Am Acad Dermatol. 2015;72(6):961-67.

6. Schaefer CP, Cappelleri JC, Cheng R, et al. Health care resource use, productivity, and costs among patients with moderate to severe plaque psoriasis in the United States. J Am Acad Dermatol. 2015;73(4):585-93.

7. Korman NJ, Zhao Y, Pike J, Roberts J, Sullivan E. Increased severity of itching, pain, and scaling in psoriasis patients is associated with increased disease severity, reduced quality of life, and reduced work productivity. Dermatol Online J. 2015;21(10). Available at: https://escholarship.org/uc/ item/1x16v3dg. Accessed April 30, 2018. 


\section{Higher Psoriasis Skin Clearance Is Associated with Lower Annual Indirect Costs in the United States: A Post Hoc Analysis from the CLEAR Study}

8. Fredriksson T, Pettersson U. Severe psoriasis—oral therapy with a new retinoid. Dermatologica. 1978;157(4):238-44.

9. Llamas-Velasco M, de la Cueva P, Notario J, Martínez-Pilar L, Martorell A, Moreno-Ramírez D. Moderate psoriasis: a proposed definition. Actas Dermosifiliogr. 2017;108(10):911-17.

10. Feldman S, Krueger G. Psoriasis assessment tools in clinical trials. Ann Rheum Dis. 2005;64(Suppl 2):ii65-ii68.

11. Puig L. PASI90 response: the new standard in therapeutic efficacy for psoriasis. J Eur Acad Dermatol Venereol. 2015;29(4):645-48.

12. Beroukhim K, Danesh M, Nguyen C, et al. A prospective, interventional assessment of the impact of ustekinumab treatment on psoriasisrelated work productivity and activity impairment. J Dermatolog Treat. 2016;27(6):552-55.

13. Blauvelt A, Reich K, Tsai T, et al. Secukinumab is superior to ustekinumab in clearing skin of subjects with moderate to severe plaque psoriasis up to 1 year: results from the CLEAR study. J Am Acad Dermatol. 2017;76(1):60-69.

14. Reilly Associates. Work Productivity and Activity Impairment Questionnaire: Specific Health Problem V2.0. August 18, 2010. Available at: http://www.reillyassociates.net/WPAI_SHP.html. Accessed May 17, 2018.
15. Reilly Associates. WPAI translations. April 16, 2018. Available at: www.reillyassociates.net/WPAI_Translations.html\#anchor_237. Accessed May 8, 2018.

16. U.S. Bureau of Labor Statistics. Table 8. Employed and unemployed fulland part-time workers by age, sex, race, and Hispanic or Latino ethnicity. 2015. Available at: http://www.bls.gov/cps/cpsaat08.pdf. Accessed April 30, 2018.

17. U.S. Bureau of Labor Statistics. National compensation survey: occupational earnings in the United States, 2010. Table 1 Summary: Mean hourly earnings and weekly hours for selected worker and establishment characteristics. May 2011. Available at: http://www.bls.gov/ncs/ocs/sp/nctbl475.pdf. Accessed April 30, 2018.

18. U.S. Bureau of Labor Statistics. Employment cost index (not seasonally adjusted): total compensation for all civilian workers in all industries and occupations, United States (national) index. 2005 to 2016. Available at: http://data.bls.gov/cgi-bin/dsrv?ci. Accessed April 30, 2018.

19. Kimball AB, Guérin A, Tsaneva M, et al. Economic burden of comorbidities in patients with psoriasis is substantial. J Eur Acad Dermatol Venereol. 2011;25(2):157-63.

20. Horn EJ, Fox KM, Patel V, Chiou CF, Dann F, Lebwohl M. Association of patient-reported psoriasis severity with income and employment. J Am Acad Dermatol. 2007;57(6):963-71. 